\title{
THE NONLOCAL NATURE OF THE SUMMABILITY OF FOURIER SERIES BY CERTAIN ABSOLUTE RIESZ METHODS
}

\author{
DAVID BORWEIN
}

(Communicated by Andrew M. Bruckner)

\begin{abstract}
It is proved that for a large class of sequences $\left\{\lambda_{n}\right\}$ the summability at a point of a Fourier series $\sum A_{n}(t)$ by the absolute Riesz method $\left|R, \lambda_{n}, 1\right|$ is not a local property of the generating function. It is also proved, inter alia, that, for every $\varepsilon>0$, the $\left|R, \lambda_{n}, 1\right|$ summability of the factored series $\sum A_{n}(t) \lambda_{n}^{-\varepsilon}$ at any point is always a local property of the generating function.
\end{abstract}

\section{INTRODUCTION}

Suppose throughout that, for $n=1,2, \ldots$,

$$
\mu_{n}>0, \quad \lambda_{n}:=\mu_{1}+\mu_{2}+\cdots+\mu_{n} \rightarrow \infty,
$$

and $s_{n}:=a_{1}+a_{2}+\cdots+a_{n}$. The series $\sum a_{n}$ is said to be summable by the absolute Riesz method $\left|R, \lambda_{n}, 1\right|$ if

$$
c(w):=\frac{1}{w} \sum_{\lambda_{n}<w}\left(w-\lambda_{n}\right) a_{n}
$$

is of bounded variation over $\left(\lambda_{1}, \infty\right)$, and it is said to be summable by the absolute weighted mean method $\left|M, \mu_{n}\right|$ if the sequence of means $\left\{t_{n}\right\}$ defined by

$$
t_{n}:=\frac{1}{\lambda_{n}} \sum_{\nu=1}^{n} \mu_{\nu} s_{\nu}
$$

is of bounded variation, that is if

$$
\sum_{n=1}^{\infty}\left|\Delta t_{n}\right|<\infty
$$

Received by the editors June 1, 1990; a summary of the contents of this paper was presented at a Conference on Approximation, Interpolation, and Summability, held in Tel-Aviv, June 4-8, 1990, in honor of Professor A. Jakimovski on the occasion of his sixty-fifth birthday.

1980 Mathematics Subject Classification (1985 Revision). Primary 40E05.

Key words and phrases. Absolute summability, Riesz, weighted mean, Fourier series, local property.

This research was supported in part by the Natural Sciences and Engineering Research Council of Canada. 
where $\Delta t_{n}:=t_{n}-t_{n+1}$. It is well known, and easily verified, that these two methods are equivalent.

Let

$$
\frac{1}{2} \alpha_{0}+\sum_{n=1}^{\infty} A_{n}(t):=\frac{1}{2} \alpha_{0}+\sum_{n=1}^{\infty}\left(\alpha_{n} \cos n t+\beta_{n} \sin n t\right)
$$

be the Fourier series generated by a periodic function $F$ with period $2 \pi$ which is Lebesgue integrable over $(-\pi, \pi)$. It is familiar that the convergence of the Fourier series at $t=x$ is a local property of $F$ (i.e. depends only on the behaviour of $F$ in an arbitrarily small neighbourhood of $x$ ), and hence the summability of the Fourier series at $t=x$ by any regular linear summability method is also a local property of $F$. On the other hand, Bosanquet and Kestleman [3] showed that the summability $|C, 1|(=|M, 1|)$ of the Fourier series at any point is not a local property of $F$, and Mohanty [7] subsequently showed that this is also the case with summability $\left|R, \lambda_{n}, 1\right|$ when $\lambda_{n}:=\ell_{k}(n)$ for $n$ sufficiently large, where

$$
\ell_{0}(x):=x \quad \text { and } \quad \ell_{k}(x):=\log \left(\ell_{k-1}(x)\right)
$$

for $k=1,2, \ldots$ and $x$ sufficiently large. Mohanty also showed that the $|R, \log n, 1|$ summability of the factored Fourier series

$$
\sum_{n=2}^{\infty} A_{n}(t) / \log n
$$

at any point is a local property of $F$, whereas the $|C, 1|$ summability of this series is not. Matsumoto [5] improved the first of these results by showing that the $|R, \log n, 1|$ summability of the series

$$
\sum_{n=3}^{\infty} A_{n}(t)(\log \log n)^{-p}, \quad p>1,
$$

at any point is a local property of $F$, and Bhatt [1] went a step further by showing that the factor $(\log \log n)^{-p}$ in the above series can be replaced by the more general factor $\gamma_{n} \log n$ where $\left\{\gamma_{n}\right\}$ is a convex sequence such that $\sum \gamma_{n} / n$ is convergent. Mishra [6] proved that if $\left\{\gamma_{n}\right\}$ is as above, and if

$$
\lambda_{n}=O\left(n \mu_{n}\right) \text { and } \lambda_{n} \Delta \mu_{n}=O\left(\mu_{n} \mu_{n+1}\right),
$$

then the summability $\left|M, \mu_{n}\right|$ of the series

$$
\sum_{n=1}^{\infty} A_{n}(t) \gamma_{n} \frac{\lambda_{n}}{n \mu_{n}}
$$

at any point is a local property of $F$. This does not directly generalize any of the above-mentioned results involving $|R, \log n, 1|$ summability since the order relations are not satisfied by $\mu_{n}:=1 / n$. Bor [2] recently showed that $\left|M, \mu_{n}\right|$ in Mishra's result can be replaced by a more general summability method $\left|M, \mu_{n}\right|_{k}$. The object of this paper is to prove the following two theorems which include most of the above-mentioned results as special cases. 
Theorem 1. Suppose that $a$ is a positive integer, and that $f$ is a positive, unbounded function with an absolutely continuous positive derivative on $\left[e^{a}, \infty\right)$ such that, on this interval,

$$
\frac{x f^{\prime}(x)}{f(x)} \text { decreases to } 0
$$

and

$$
x f^{\prime \prime}(x)=O\left(f^{\prime}(x)\right)
$$

Suppose also that

$$
\lambda_{n}:=f\left(e^{n}\right) \text { for }+n \geq a,
$$

and that $0<\alpha<\beta<2 \pi$. Then there is a function $F$, Lebesgue integrable over $(\alpha, \beta)$ and zero in the remainder of $(0,2 \pi)$, whose Fourier series is not summable $\left|R, \lambda_{n}, 1\right|$ at $t=0$.

This shows that, subject to the hypotheses of the theorem, the summability $\left|R, \lambda_{n}, 1\right|$ of a Fourier series at any point is not a local property of its generating function. Since the hypotheses are satisfied by $f(x):=\ell_{k}(x)$ for $k=1,2, \ldots$, Bosanquet and Kestleman's result, and also Mohanty's result, on the nonlocal nature of the summability of a Fourier series by certain absolute methods are special cases of Theorem 1.

Theorem 2. Suppose that the sequence $\left\{c_{n}\right\}$ is such that

$$
\sum_{n=1}^{\infty} \frac{\mu_{n}}{\lambda_{n}}\left|c_{n}\right|<\infty
$$

and

$$
\sum_{n=1}^{\infty}\left|\Delta c_{n}\right|<\infty
$$

Then the summability $\left|R, \lambda_{n}, 1\right|$ of the factored Fourier series

$$
\sum_{n=1}^{\infty} A_{n}(t) c_{n}
$$

at any point is a local property of the generating function $F$.

This theorem generalizes Bhatt's above-mentioned result, since it is known (see [1] for references) that if $\left\{\gamma_{n}\right\}$ is a convex sequence such that $\sum \gamma_{n} / n$ is convergent, then

$$
\gamma_{n} \geq \gamma_{n+1} \geq 0 \text { and } \sum_{n=1}^{\infty} \log n \Delta \gamma_{n}<\infty,
$$

and so (4) and (5) are satisfied by $\mu_{n}:=1 / n, c_{n}:=\gamma_{n} \log n$. Since, by Dini's theorem, $\sum \mu_{n} \lambda_{n}^{-1-\varepsilon}$ is convergent whenever $\varepsilon>0$, we have the following corollary of Theorem 2 . 
Corollary. For $\varepsilon>0$, the summability $\left|R, \lambda_{n}, 1\right|$ of the factored Fourier series

$$
\sum_{n=1}^{\infty} A_{n}(t) \lambda_{n}^{-\varepsilon}
$$

at any point is a local property of the generating function $F$.

\section{Preliminary Results}

Lemma 1. Suppose that the function $f$ satisfies the conditions of Theorem 1 and that $g$ is its inverse function. Let $\lambda_{n}:=f\left(e^{n}\right)$, and

$$
h(w):=\frac{g(w)}{w g^{\prime}(w)} \quad \text { on }+[b, \infty)
$$

where $b:=f\left(e^{a}\right)$. Then

$$
h(w) \text { decreases to } 0
$$

and

$$
w h^{\prime}(w)=O(1)
$$

on $[b, \infty)$. Further,

$$
\sum_{n=a}^{\infty} h\left(\lambda_{n}\right)=\infty
$$

Finally, if $\sum a_{n}$ is summable $\left|R, \lambda_{n}, 1\right|$, then $\sum a_{n} h\left(\lambda_{n}\right)$ is absolutely convergent.

Proof. Let $w=f(x)$ for $x \geq e^{a}$. Then $x=g(w)$ and $1=g^{\prime}(w) f^{\prime}(x)$, whence $h(w)=x f^{\prime}(x) / f(x)$. Thus (6) is a consequence of (1). Next,

$$
0=g^{\prime \prime}(w) f^{\prime}(x)^{2}+g^{\prime}(w) f^{\prime \prime}(x)=\frac{g^{\prime \prime}(w)}{g^{\prime}(w)} f^{\prime}(x)+g^{\prime}(w) f^{\prime \prime}(x)
$$

so that

$$
\begin{aligned}
w h^{\prime}(w) & =w h(w)\left(\frac{g^{\prime}(w)}{g(w)}-\frac{1}{w}-\frac{g^{\prime \prime}(w)}{g^{\prime}(w)}\right)=1-h(w)-\frac{w g(w) g^{\prime \prime}(w)}{g^{\prime}(w)^{2}} \\
& =1-h(w)+\frac{x f^{\prime \prime}(x)}{f^{\prime}(x)} .
\end{aligned}
$$

Hence (7) is a consequence of (2) and (6).

In order to establish (8), let $\lambda(x):=f\left(e^{x}\right)$, so that $\lambda_{n}=\lambda(n)$. Then, for $x \geq a$, we have $g(\lambda(x))=e^{x}$ so that $g^{\prime}(\lambda(x)) \lambda^{\prime}(x)=e^{x}$, and hence

$$
h(\lambda(x))=\frac{g(\lambda(x))}{\lambda(x) g^{\prime}(\lambda(x))}=\frac{\lambda^{\prime}(x)}{\lambda(x)}
$$

Therefore

$$
\int_{a}^{y} h(\lambda(x)) d x=\log (\lambda(y))-\log (\lambda(a)) \rightarrow \infty \quad \text { as } y \rightarrow \infty .
$$

Conclusion (8) follows, by the integral test.

Suppose now that $\sum a_{n}$ is $\left|R, \lambda_{n}, 1\right|$ summable. Since $g\left(\lambda_{n}\right)=e^{n}$, it follows from (6) and (7), by a result due to Dikshit [4], that $\sum a_{n} h\left(\lambda_{n}\right)$ is 
$\left|R, e^{n}, 1\right|$ summable, and Mohanty [8, Lemma 4] has shown this to be equivalent to $\sum a_{n} h\left(\lambda_{n}\right)$ being absolutely convergent.

Lemma 2. Suppose that the sequence $\left\{c_{n}\right\}$ satisfies conditions (4) and (5) of Theorem 2, and that $\left\{s_{n}\right\}$ is bounded. Then

$$
\sum_{n=1}^{\infty} a_{n} c_{n}
$$

is summable $\left|R, \lambda_{n}, 1\right|$.

Proof. Let $\left\{T_{n}\right\}$ be the sequence of $\left(M, \mu_{n}\right)$ means of series (9), that is

$$
T_{n}:=\frac{1}{\lambda_{n}} \sum_{\nu=1}^{n} \mu_{\nu} \sum_{r=1}^{\nu} a_{r} c_{r}=\frac{1}{\lambda_{n}} \sum_{r=1}^{n}\left(\lambda_{n}-\lambda_{r-1}\right) a_{r} c_{r}
$$

where $\lambda_{0}:=0$. We wish to show that

$$
\sum_{n=1}^{\infty}\left|\Delta t_{n}\right|<\infty
$$

We have that

$$
\begin{aligned}
T_{n+1}-T_{n} & =\frac{\mu_{n+1}}{\lambda_{n} \lambda_{n+1}} \sum_{r=1}^{n+1} \lambda_{r-1} a_{r} c_{r} \\
& =\frac{\mu_{n+1}}{\lambda_{n} \lambda_{n+1}} \sum_{r=1}^{n} s_{r}\left(\lambda_{r} \Delta c_{r}-\mu_{r} c_{r}\right)+\frac{\mu_{n+1} c_{n+1} s_{n+1}}{\lambda_{n+1}} .
\end{aligned}
$$

Hence, if we suppose that $\left|s_{n}\right| \leq 1$, as we may without loss of generality, we see that

$$
\left|\Delta t_{n}\right| \leq \frac{\mu_{n+1}}{\lambda_{n} \lambda_{n+1}} \sum_{r=1}^{n}\left(\lambda_{r}\left|\Delta c_{r}\right|+\mu_{r}\left|c_{r}\right|\right)+\frac{\mu_{n+1}}{\lambda_{n+1}}\left|c_{n+1}\right|,
$$

and so

$$
\begin{aligned}
\sum_{n=1}^{\infty}\left|\Delta t_{n}\right| & \leq \sum_{r=1}^{\infty}\left(\lambda_{r}\left|\Delta c_{r}\right|+\mu_{r}\left|c_{r}\right|\right) \sum_{n=r}^{\infty} \frac{\mu_{n+1}}{\lambda_{n} \lambda_{n+1}}+\sum_{n=2}^{\infty} \frac{\mu_{n}}{\lambda_{n}}\left|c_{n}\right| \\
& =\sum_{r=1}^{\infty}\left(\left|\Delta c_{r}\right|+\frac{\mu_{r}}{\lambda_{r}}\left|c_{r}\right|\right)+\sum_{n=2}^{\infty} \frac{\mu_{n}}{\lambda_{n}}\left|c_{n}\right|<\infty,
\end{aligned}
$$

by (4) and (5).

\section{Proofs of the theorems}

Proof of Theorem 1. By Lemma 1, it suffices to show that there is a function $F$, Lebesgue integrable over $(\alpha, \beta)$, such that

$$
\sum_{n=a}^{\infty}\left|\int_{\alpha}^{\beta} h\left(\lambda_{n}\right) F(t) \cos n t d t\right|=\infty,
$$


where the function $h$ is as in the lemma. For $0<t<2 \pi, t \neq \pi$, we have that

$$
\begin{aligned}
\sum_{n=a}^{\infty} h\left(\lambda_{n}\right)|\cos n t| & \geq \sum_{n=a}^{\infty} h\left(\lambda_{n}\right) \cos ^{2} n t \\
& \geq \frac{1}{2} \sum_{n=a}^{\infty} h\left(\lambda_{n}\right)-\frac{1}{2}\left|\sum_{n=a}^{\infty} h\left(\lambda_{n}\right) \cos 2 n t\right|=\infty
\end{aligned}
$$

by Lemma 1 , the final sum being convergent because the sequence $\left\{h\left(\lambda_{n}\right)\right\}$ decreases to 0 . The required result now follows from a theorem due to Bosanquet and Kestleman [3, Theorem 1].

Proof of Theorem 2. Since the convergence of the Fourier series at a point is a local property of its generating function $F$, Theorem 2 follows immediately from Lemma 2.

Remark (added November 9, 1990). After this paper was accepted for publication I found out that Theorem 2 is in fact a special case of Theorem 3 in S. Baron's paper, Local property of absolute summability of a Fourier series and the conjugate series, Tartu Riikl. Ül. Toimetised Vih. 253 (1970), 212-228. My proof, however, is somewhat simpler and more direct than Baron's, partly because he deals with more general summability methods.

\section{REFERENCES}

1. S. N. Bhatt, An aspect of local property of $|R, \log n, 1|$ summability of factored Fourier series, Proc. Nat. Inst. Sci. India 26 (1960), 69-73.

2. H. Bor, Local property of $\left|\bar{N}, p_{n}\right|_{k}$ summability of factored Fourier series, Bull. Inst. Math. Acad. Sinica 17 (1989), 165-170.

3. L. S. Bosanquet and H. Kestleman, The absolute convergence of series of integrals, Proc. London Math. Soc. (2) 45 (1939), 88-97.

4. G. D. Dikshit, On the absolute Riesz summability factors of infinite series (I), Indian J. Math. 1 (1958), 33-40.

5. K. Matsumoto, Local property of summability $\left|R, \lambda_{n}, 1\right|$, Tôhoku Math. J. (2) 8 (1956), 114-124.

6. K. N. Mishra, Multipliers jor $\left|\bar{N}, p_{n}\right|$ summability of Fourier series, Bull. Inst. Math. Acad. Sinica 14 (1986), 431-438.

7. R. Mohanty, On the summability $|R, \log \omega, 1|$ of a Fourier series, J. London Math. Soc. 25 (1950), 67-72.

8. Math. Soc. 52 (1951), 295-321.

Department of Mathematics, University of Western Ontario, London, Ontario, N6A 5B7 CANADA 